\title{
EDITORIAL
}

\section{In search of a unifying diagnosis}

Pediatric Research (2021) 89:251; https://doi.org/10.1038/s41390020-01316-2

Generations of pediatricians have grouped signs and symptoms in the quest for a unifying diagnosis. Coupled with this approach, children grouped together under related diagnoses have received similar interventions and treatment plans. Understandably, individual responses to therapies vary, but the "whys" and "hows" that underscore these individual differences are not well understood. The resulting paradigm deconstructs the practice of grouping and recognizes that individuals often require personalized approaches to sustain health and navigate treatment options.

A key intersection for personalized or precision medicine is the influence of environmental factors and circumstances, including where children live, family income and education, food security, and social support. A growing body of evidence not only demonstrates a strong connection between these "social determinants" and child health, but also suggests how much weight individual factors may exert on particular diseases or response to interventions and therapies. These novel and measurable connections provide practitioners with the knowledge to cater their healthcare to individual(s) and select one intervention or therapy over another. Truly, pediatricians and stakeholders in child health are sitting at the precipice of a new era of medicine gazing on a nuanced and likely more effective means of caring for children.
In this Annual Review Issue of Pediatric Research, leaders in child health, policy, and research provide a vivid portrayal of the interaction between social determinants of health and precision medicine. Novel questions such as "Do the nutritional needs of preterm infants differ by gender?" and "How might maternal diet influence the microbiome of their offspring?" represent the next phase of scientific endeavors. Moreover, questions including "Does race, gender, or nationality delay diagnosis in difficult cases?" and "Why do ethnic disparities in rates of human milk feeding exist?" provide insight into how pediatricians actually personalize their medicine for the child on the exam table. The Editors of Pediatric Research invite you to read and consider how this Annual Review Issue might equip you with new tools to care for children and provide you with new opportunities to "see" the individual within the group.

Brian Stansfield $\left(D^{1,2}\right.$, Eleanor Molloy ${ }^{3,4,5}$ and Cynthia Bearer ${ }^{6,7}$ ${ }^{1}$ Neonatal/Perinatal Medicine, Children's Hospital of Georgia, Augusta, GA, USA; ${ }^{2}$ Pediatrics and Vascular Biology Center, Medical College of Georgia at Augusta University, 1120 15th St Augusta,

Augusta, GA 30912, USA; ${ }^{3}$ Paediatrics and Child Health, Trinity Translational Medicine Institute, Trinity College, University of Dublin, Dublin, Ireland; ${ }^{4}$ Children's Health Ireland at Tallaght and Crumlin, Dublin, Ireland; ${ }^{5}$ Coombe Women's and Infants University Hospital, Dublin, Ireland; ${ }^{6}$ Division of Neonatology, UH Rainbow Babies \& Children's Hospital, Cleveland, $\mathrm{OH}$, USA and 'Pediatrics, Case Western Reserve University School of Medicine, Cleveland, $\mathrm{OH}$, USA Correspondence: Brian Stansfield (BSTANSFIELD@augusta.edu) 